Check for updates

Cite this: Sustainable Energy Fuels, 2020, 4, 852

Received 30th July 2019

Accepted 1st November 2019

DOI: $10.1039 / c 9 s e 00948 e$

rsc.li/sustainable-energy

\section{Roadmap for cost-effective, commercially-viable perovskite silicon tandems for the current and future PV market $\uparrow$}

\author{
Sarah E. Sofia, (D)*a Hao Wang, ${ }^{\text {b }}$ Annalisa Bruno, (D) ${ }^{\mathrm{b}}$ Jose Luis Cruz-Campa, ${ }^{\mathrm{c}}$ \\ Tonio Buonassisi*a and Ian Marius Peters ${ }^{* a}$
}

\begin{abstract}
A techno-economic analysis of perovskite-silicon tandem solar modules is presented, outlining the most viable pathway for designing cost-effective, commercially viable tandems. We explore the costperformance trade-off for silicon bottom cells in perovskite-silicon tandems, and evaluate the potential of using low-cost, lower-efficiency silicon bottom cells, on the basis of levelized cost of electricity (LCOE), compared to the higher-efficiency, higher-cost bottom cells that have been the primary focus of most perovskite-silicon tandem research efforts. We fabricate a cost-effective four-terminal siliconperovskite tandem using a low-cost multicrystalline bottom cell and calculate the device LCOE. We then extend this analysis by modeling performance and LCOE of similar tandems instead using high-efficiency silicon bottom cells, enabling direct comparison of a low-cost and a high-efficiency tandem. Lastly parametric analyses are performed to more broadly examine the bottom-cell cost-performance tradeoff. We show that low-cost silicon, even at the detriment of efficiency, is the more likely path to make perovskite-silicon tandems commercially viable and enable future reductions in LCOE, given both current and near-future silicon technology. We lay out a clear economic motivation for pursuing lowcost silicon bottom cells in perovskite-silicon tandems, showing that they can achieve a $15-20 \%$ relative LCOE reduction compared to the single-junction sub-cells. This is a $2-3$ times greater relative LCOE reduction compared with using high-efficiency silicon. Furthermore, we show that the advantage of using low-cost silicon bottom cells is robust to and benefits from expected market trends, such as falling system costs and advanced, low-cost manufacturing. This work provides a clear pathway to costeffective tandems, outlines the benefits for existing multicrystalline silicon manufacturers to investing in tandem development, and points out a clear mismatch between commercial viability and current research efforts.
\end{abstract}

\section{Introduction and background}

Tandem architectures present a path to reach high solar cell efficiency with the potential to surpass traditional, singlejunction (SJ) limits. Increasing efficiency is one of the strongest technical drivers to reduce the cost-per-watt of solar technology, ${ }^{1,2}$ so as efficiencies of current mainstream technology approach the theoretical limit, novel technologies are needed to continue to improve efficiency and drive down the cost of solar electricity. This has led to significant interest in the development of tandem solar cells for terrestrial applications. A primary focus of recent tandem research efforts has been perovskite-on-silicon tandems, because (1) the tunable bandgap

${ }^{a}$ Massachusetts Institute of Technology, Cambridge, MA, 02139, USA

${ }^{b}$ Nanyang Technology University, 50 Nanyang Ave, Singapore

'1366 Technologies, 6 Preston Court, Bedford, MA 01730, USA

$\dagger$ Electronic supplementary information (ESI) available. See DOI: 10.1039/c9se00948e of perovskites (typically from 1.5 to $2.2 \mathrm{eV}$ ) allows the creation of a potentially ideal bandgap top cell to couple with silicon, and (2) this material pairing enables leveraging the $>95 \%$ market share currently held by silicon while utilizing the low-cost,,4 rapidly developing perovskite technology, together holding the promise to make tandems economically superior.

While perovskite-silicon tandems have these advantages, there are challenges to making a cost-effective tandem. Though tandems can achieve higher efficiencies, they are also more expensive and complex to manufacture. The efficiency gain of the tandem relative to the comprising single-junction (SJ) cells must be sufficient to justify the added manufacturing cost of integrating an additional sub-cell, such that the tandem is more economical than either comprising sub-cell on its own as a SJ. Generally, sub-cells that have similar SJ efficiencies and areal cell costs are most likely to produce a cost-effective tandem device, a property referred to as the 'marriage of equals'. ${ }^{5}$ This balance is difficult for perovskite-silicon tandems since they feature two technologies with very different manufacturing 
approaches: perovskite using solution-processed thin-film deposition and silicon being a wafer-based technology. Thus, this tandem potentially combines a very low cost material with low capital expenditure (CapEx) with silicon solar cells that are more expensive and fairly CapEx intensive to manufacture. While a recent techno-economic analysis shows that siliconbased tandems are likely to be a financially viable technology in the future solar market, ${ }^{6}$ there has been little exploration into what makes a silicon-based tandem optimally cost-competitive.

There are two clear design directions that can be taken for the silicon bottom cell in order to make a cost-effective perovskite-silicon tandem: (1) reducing the cost of the silicon bottom cell as much as possible to minimize its financial impact on the cell and reduce the overall module cost, or (2) maximizing the power output of the bottom cell to help justify its inclusion in the device stack and produce a higher efficiency module. The low-cost option has a clear advantage in promoting similar costs between the two sub-cells, as advised by the 'marriage of equals' principal, while the high-efficiency option maximally leverages efficiency improvement as a strong driver to reduce the cost-per-watt on a module and system level. Of course, achieving both a low-cost and high-efficiency cell would be ideal, there is generally a trade-off between efficiency and cost, since higher efficiency cell technology typically requires better material quality and more complex device architecture which increase manufacturing cost.

Many impressive perovskite-silicon tandem results have been achieved: the current record four-terminal (4T) (mechanicallystacked, electrically independent sub-cells) efficiency record is $26.4 \%,{ }^{7}$ and the current two-terminal (2T) (monolithically integrated, series-connected sub-cells) efficiency record is $25.2 \% .^{8}$ (A $28 \%$ efficiency $2 \mathrm{~T}$ device has been reported, ${ }^{9}$ however no device details are published). These results, like many other published results, focus on maximizing efficiency by utilizing high-cost, monocrystalline silicon cells for the bottom cells with 1-sun efficiencies of 23.9 and $22.6 \%,{ }^{8}$ respectively. While demonstrating high efficiency devices is worthwhile to show the potential of this technology and push toward higher performance devices, the use of high-efficiency, high-cost bottom cells may not be the best path to cost-effective tandems, as this further exacerbates the cost discrepancy between the low-cost perovskite top cell and the more expensive, CapEx-intensive silicon bottom cell.

Furthermore, the bottom cell typically produces less than half of the total energy generated by a tandem, so the bottom cell performance has less impact on the overall tandem efficiency than the top cell. This further suggests the possible advantage of low-cost, multicrystalline silicon wafers, potentially employing low-cost crystallization techniques such as ingot casting and kerfless or direct-wafering techniques, for use in tandem applications rather than a high-cost, higher efficiency cell: in addition to being a better 'marriage of equals' match for perovskite cells, (1) the lower cost cell can more easily be justified by the small fraction of the tandem power they produce, (2) the tandem is less sensitive to variation in performance of the bottom cell due to their smaller role, making high-performance less valuable for a bottom cell. This is further motivated by multicrystalline silicon's $62 \%$ market share of solar production ${ }^{10}$ and recent news of kerfless Direct Wafer® technology transitioning into production scale. ${ }^{11}$ Despite these indications that low-cost silicon is likely more viable for designing a cost-effective perovskite-silicon tandem, there have been almost no research efforts looking at multicrystalline silicon-perovskite tandems.

To explore whether low-cost silicon bottom cells do, in fact, have an economic advantage and more broadly understand how to best design these devices and direct research efforts, we use a techno-economic approach to explore the financial viability of perovskite-silicon tandem solar modules, and aim define the economically-best roadmap for the development of perovskitesilicon tandems. We compare two primary tandems: (1) the high efficiency tandem: a perovskite-silicon tandem utilizing a highefficiency, high-cost heterojunction with intrinsic thin layer (HIT) monocrystalline silicon solar cell, and (2) the low-cost tandem: a perovskite-silicon tandem employing a low-cost, lower efficiency multicrystalline passivated emitter and rear cell (PERC) silicon solar cell. The explored tandems and SJ device architecture are shown in Fig. 1. First, we fabricate a 'low-cost' tandem using an industrially processed PERC mc-Si bottom cell, demonstrating the viability of a perovskite based tandem using a multicrystalline silicon bottom cell. We additionally model potential improvements to this device, as well as compare to a modelled highefficiency tandem. Levelized cost of electricity (LCOE) is used to evaluate the relative viability of these two tandems, combining module manufacturing costs and energy yield calculations and considering current and potential future cost scenarios for both residential and utility scale systems. We then extend this approach to perform a few parametric analyses to understand the relative merits and potential viability of each tandem. By doing so, we propose a framework to understanding when to push toward maximizing the absolute device efficiency versus leveraging the tandem architecture to boost the efficiency of low-cost solar technology and thus making cheap solar cells viable to compete with high efficiency SJ technology and lowering the cost of solar electricity.

\section{Methodology}

\section{A. Yield calculations and architectures}

Energy yield calculations are performed using the method presented in ref. 12 and 13, employing hourly time-resolved, location-specific spectra generated using the Simple Model for Atmospheric Radiative Transfer of Sunshine (SMARTS) from the National Renewable Energy Laboratory ${ }^{14,15}$. We perform all calculations for three, climactically distinct locations: Albuquerque, NM (dry), Rapid City, ND (temperate), and Miami, FL (humid). We present only calculations for Albuquerque, NM in the main manuscript, as the results across different locations are very similar however calculations using the other two locations are included in the ESI. $\dagger$

\section{B. LCOE calculations and cost model}

The figure of merit used to evaluate each device architecture is the levelized cost of electricity (LCOE). LCOE is calculated by the equation, ${ }^{16}$ 
(a) 'Low-cost' silicon SJ cell

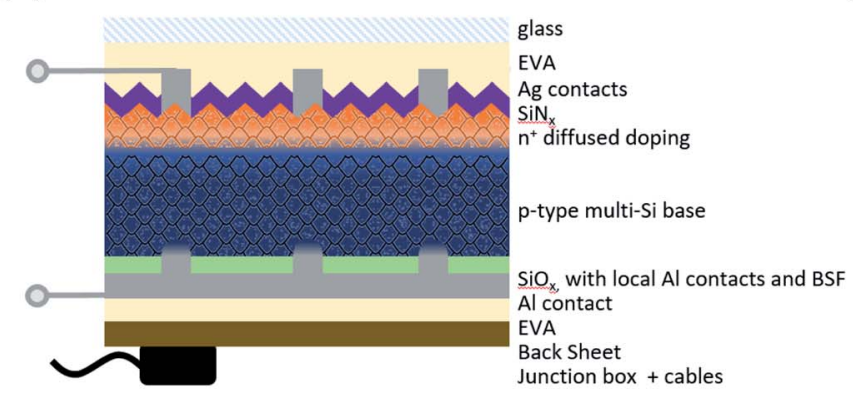

(b) 'High-efficiency' silicon SJ cell

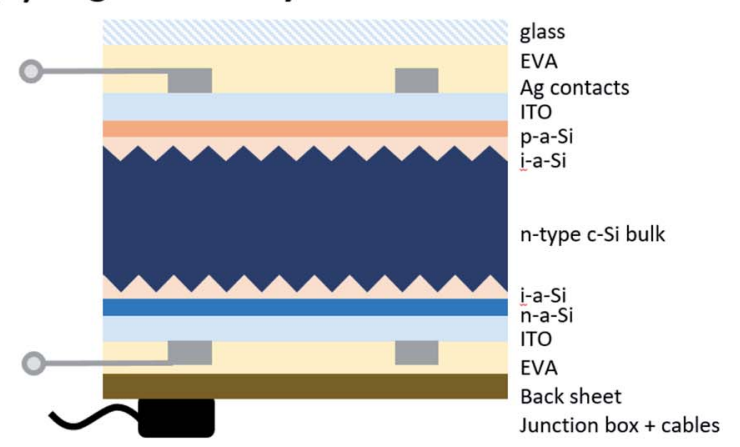

(c) 'Low-cost' 4T tandem

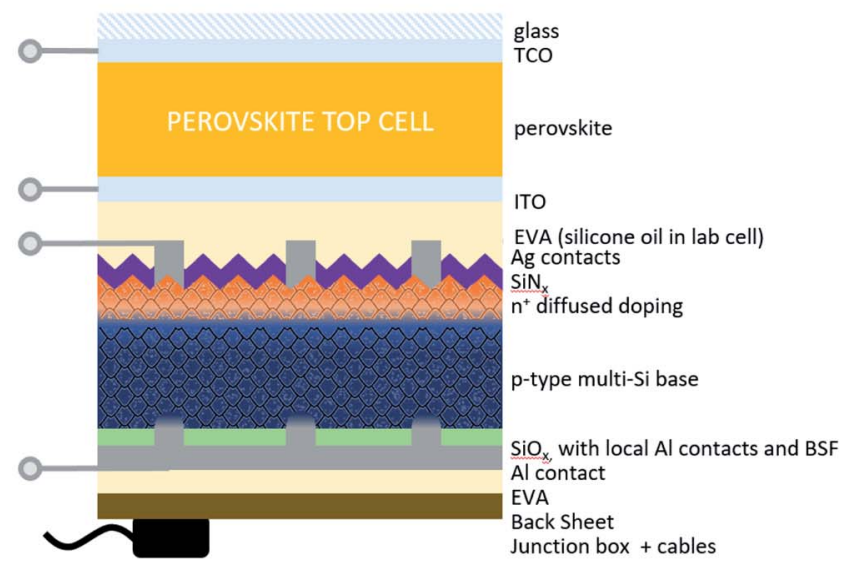

(d) 'High-efficiency' 4T tandem

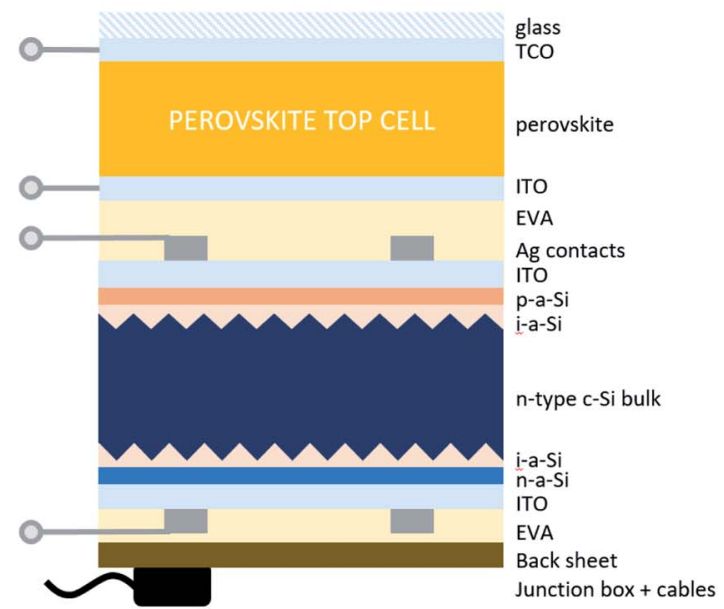

Fig. 1 Schematic of the two silicon single-junction modules and the two 4T tandems. These schematics indicate the full stack used for the module manufacturing.

$$
\mathrm{LCOE}=\frac{\text { total lifetime cost }}{\text { total lifetime electricity production }}=\frac{I+\sum_{i=0}^{N} \frac{\mathrm{OM}}{1+r}}{\sum_{i=0}^{N} \frac{E(1-d)^{i}}{1+r}}
$$

where $I$ is the total initial investment to install the PV system (including cost of PV modules, racking, interconnects, labor, and permits), OM is the annual cost for operation and maintenance, $E$ is the (un-degraded) annual energy output by the system as electricity, $N$ is the system lifetime in years, $d$ is the annual module efficiency degradation rate, and $r$ is the nominal discount rate. The values of the financial input parameters are given in Table 1 . An annual degradation rate of $0.5 \%$, relative, is assumed for both the silicon and perovskite cells. This is consistent with current silicon degradation rates, ${ }^{\mathbf{1 7}}$ while perovskite have demonstrated significant stability issues. ${ }^{18}$ Rather, the current longest reported stable lifetime of a perovskite solar cell is only 1 year. ${ }^{19}$ For this analysis, however, we assume continued progress will be made on perovskite cells, as perovskite SJ cells and perovskite-silicon tandems will not be viable for long-term, grid-connected applications unless perovskite solar cells achieve similar degradation rates to silicon.
We define a manufacturing process flow for each tandem module and used this to developed a bottom-up module manufacturing cost model for the two four-terminal tandems based on existing cost models ${ }^{\mathbf{3 , 4 1 3 , 2 0}}$. Using this model, we calculated the minimum sustainable price (MSP) assuming straight-line depreciation and a $14 \%$ internal rate of return (IRR). ${ }^{1}$ The tandem and SJ module costs and MSPs are given in Table 2. See ESI $\dagger$ for a more detailed cost breakdown.

Lastly, to calculate the total cost of the installed system, we broke down PV system cost data from ${ }^{21}$ into area, power, and project dependent costs ${ }^{22}$ to project system installation costs for a given residential or utility PV system (Table 1). We also consider a hypothetical future reduced system cost scenario, presented in ref. 13, based on the costs presented in ref. 23 that aim to meet the United State Department of Energy Sunshot goals. We assume a constant module area of $35.9 \mathrm{~m}^{2}$ and $0.6 \mathrm{~km}^{2}$ for all residential and utility systems, respectively.

To examine the benefit of the tandem architecture compared to the single-junction sub-cells on their own, we use the 'relative tandem advantage' as a metric. The relative tandem advantage is defined as, 
Table 1 Financial and system parameters and system installation costs used to calculate LCOE for residential and utility scale systems based on both current (2018) costs and a potential reduced system cost scenario representing a potential future cost breakdown (future reduced)

System costs and LCOE input parameters

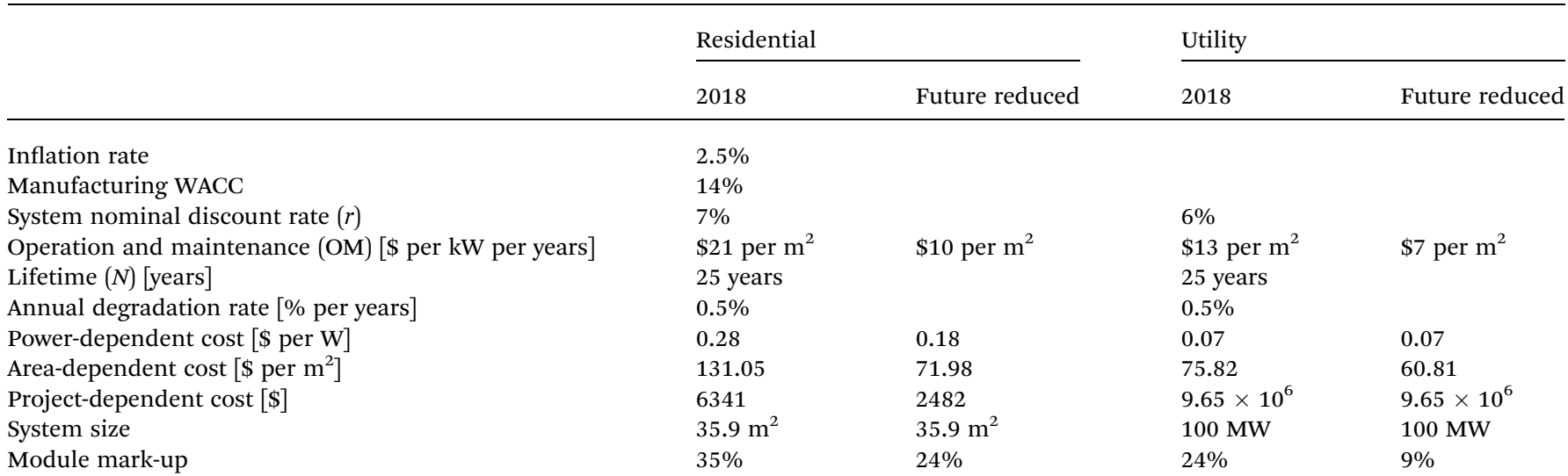

Table 2 Device manufacturing minimum sustainable prices, in U.S. dollars per square-meter. See ESI for a more detailed breakdown

Module manufacturing cost and prices

Module price $\left[\mathrm{S}\right.$ per $\left.\mathrm{m}^{2}\right]$

'High-efficiency' Si SJ cell: HIT c-Si SJ

'Low-cost' Si cell: PERC mc-Si SJ

Perovskite SJ

'High-efficiency' 4T tandem

'Low-cost' 4T tandem

$\$ 79.31$ per $\mathrm{m}^{2}$

$\$ 62.90$ per $\mathrm{m}^{2}$

$\$ 38.69$ per $\mathrm{m}^{2}$

$\$ 96.88$ per $\mathrm{m}^{2}$

$\$ 80.25$ per $\mathrm{m}^{2}$

Relative tandem advantage $=$

$$
\frac{\min \left(\mathrm{LCOE}_{\mathrm{Si}}, \mathrm{LCOE}_{\text {perov. }}\right)-\mathrm{LCOE}_{4 \mathrm{~T}}}{\min \left(\mathrm{LCOE}_{\mathrm{Si}}, \mathrm{LCOE}_{\text {perov. }}\right)}
$$

showing when the relative reduction in LCOE for a tandem compared to the lowest LCOE sub-cell as a single-junction is largest.

\section{Results and discussion}

\section{A. Device fabrication and modeling}

We first fabricated the low-cost tandem, using an industrially processed PERC multicrystalline silicon solar cell from an industrial partner, laser cut to $0.8 \mathrm{~cm}^{2}$, and coated in $36 \mathrm{~nm}$ $\mathrm{Al}_{2} \mathrm{O}_{3}$ by ALD for edge passivation. The small-area, masked $(0.72$ $\mathrm{cm}^{2}$ aperture) cell efficiency was $17.2 \%$. The open-circuit voltage $\left(V_{\mathrm{OC}}\right)$ and the efficiency of the cut, small area cell was significantly reduced compared to the full, 6 inch wafer measurement, with the efficiency dropping from $19 \%$ to $17.2 \%$ and the $V_{\mathrm{OC}}$ dropping from 0.65 to $0.61 \mathrm{~V}$ (with edge-passivation). This is believed to be due primary to (1) edge recombination becoming significant for a small area device, (2) dark-current due to a small masked area (about $1 \mathrm{~mm}$ wide strip on a side, $10 \%$ of wafer) reduction in shunt-resistance due to the wafer cutting. This cell was aligned and measured under a $1.7 \mathrm{eV}$ semitransparent perovskite top-cell, fabricated in substrate configuration, with an efficiency of $13.1 \%$. The resulting fourterminal, mechanically-stacked tandem had a total efficiency of $19.1 \%$, with $6 \%$ produced by the bottom cell (Fig. 2 and Table 3). While this device performance is not record breaking, it demonstrates the potential of a low-cost, industrially-relevant mc-Si cell to act as a decent bottom cell and create a tandem that surpasses the sub-cell efficiencies.

Using the measured external quantum efficiency (EQE) and current-voltage $(J V)$ curves, along with the cost models, we compute the LCOE for the SJ sub-cells and the tandem for current and future residential and utility scale systems (Table 3). We see that even our prototype tandem has the lowest LCOE in both residential cases. Our fabricated tandem, however, is far from ideal, and one clear path for improvement is to increase the perovskite top cell efficiency. Reported values up of to $16 \%$ for semi-transparent ${ }^{24}$ and around $20 \%$ for non-transparent cells $^{25,26}$ of similar bandgap have been demonstrated, compared to our $13.1 \%$ cell. Additionally, the losses due to the small area such as edge effects and dark current from masking hurt the tandem performance and unrealistic for a full-size module. Thus, to explore the future potential of this tandem device, we model the performance and calculate the LCOE of the tandem and single-junction with bottom cell parameters reflecting full area silicon cell (see Table 4) and for the case of an $18 \%$ efficient perovskite cell with an average transmission of $80 \%$ (Table 3 , second column). The transmission curve is modeled by scaling-up the sub-bandgap portion of the measured transmission curve of our experimental perovskite cell. See ESI $\dagger$ for more detail on the 18\% top-cell. Further improvements to tandem bottom cell performance could be made by tailoring the silicon bottom cell device architecture for application as a tandem bottom cell, such as through optimizing anti-reflection coatings and bulk-resistivity for longwavelength response. For this work, however, we simply show the potential of commercial silicon cells as a drop-in solution for tandems, without processing changes.

Lastly, in order to compare low-cost silicon-based tandems to tandems with a higher-efficiency silicon bottom cell, we 


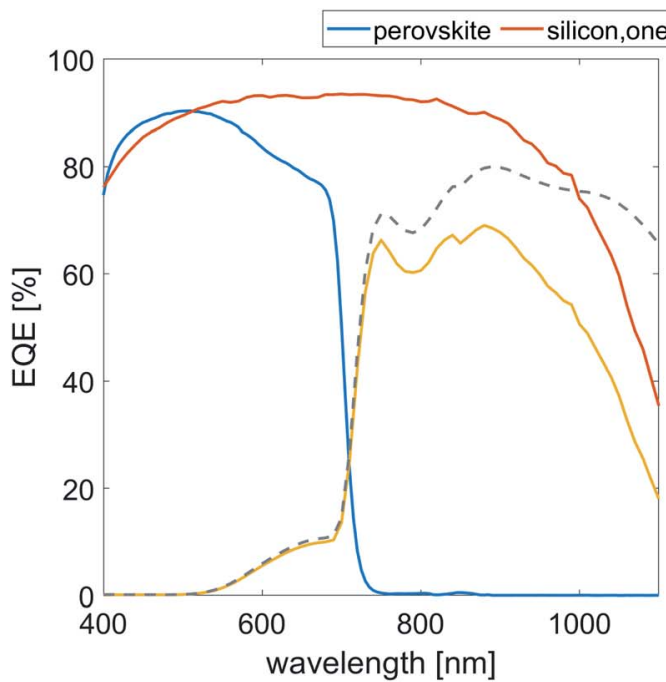

(a)

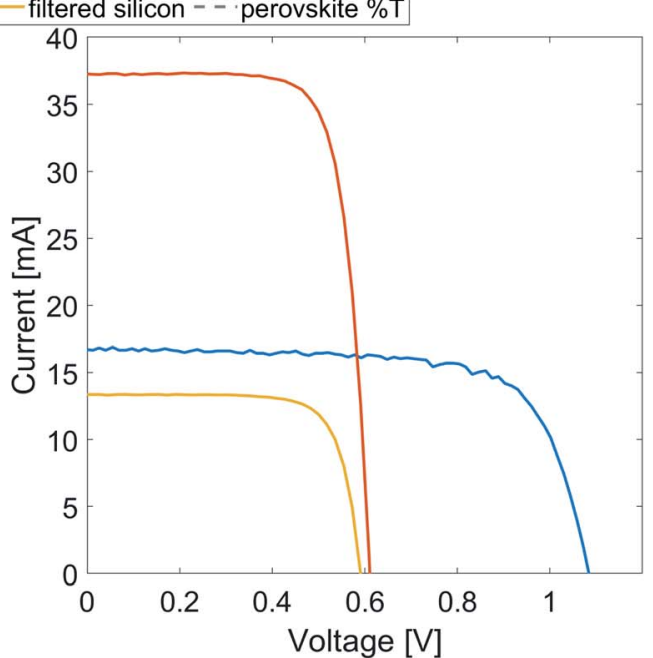

(b)

Fig. 2 (a) External quantum efficiency (EQE) and (b) current-voltage (JV) curve of the perovskite top cell, the silicon bottom cell under one-sun illumination, and the silicon bottom cell under one-sun filtered by the perovskite top cell.

model the performance of the tandems using the same two perovskite top cells $(13.1 \%$ and $18 \%$ efficient) but with a highefficiency, monocrystalline HIT bottom cell. We use published, record-efficiency front-and-back-contact HIT monocrystalline Si cell EQE and JV parameters ${ }^{27,28}$ with modeled added module losses (Table 4) and calculate the bottom cell $\mathrm{EQE}$ as the product of the transmission curve and reported onesun EQE. We then compute the LCOE for each of these modeled high-efficiency silicon-based tandems. All results are summarized in Table 3, totaling four devices comprised of different pairings of two perovskite top cells and two silicon bottom cells. We refer to the two tandems using mc-Si as 'low-cost' and the two tandems using the HIT monocrystalline Si cells as 'highefficiency'.

We see that for both residential system scenarios, the experimental low-cost tandem with the $13.1 \%$ top cell is costeffective relative to its sub-cells, and the low-cost tandem with the $18 \%$ top cell is cost-effective relative to its sub-cells in all four scenarios. The high-efficiency tandem with the $18 \%$ top cell is cost-effective in 2018 and reduced residential as well as 2018 utility systems, while the high-efficiency tandem with the $13.1 \%$ top cell is not cost-effective in any of the given scenarios. For utility scale systems, all tandems using the $13.1 \%$ top cell have an LCOE higher than both sub-cells, favoring the silicon SJs, while all tandems with the $18 \%$ top-cell have an LCOE lower

Table 3 Efficiency for each considered measured or modelled tandem and the calculate LCOE. The LCOE is reported in the order: Residential 2018 (Res 2018), Residential Reduced System Cost Scenario (SS Res), Utility 2018 (2018 Util), Utility Reduced System Cost Scenario (SS Util). The lowest LCOE among the tandem and sub-cell single-junctions is in bold, and the lowest LCOE for each of the four system scenarios among all tandems and single-junctions across all four devices is underlined. See Table 4 for further detail on device efficiencies and IV parameters

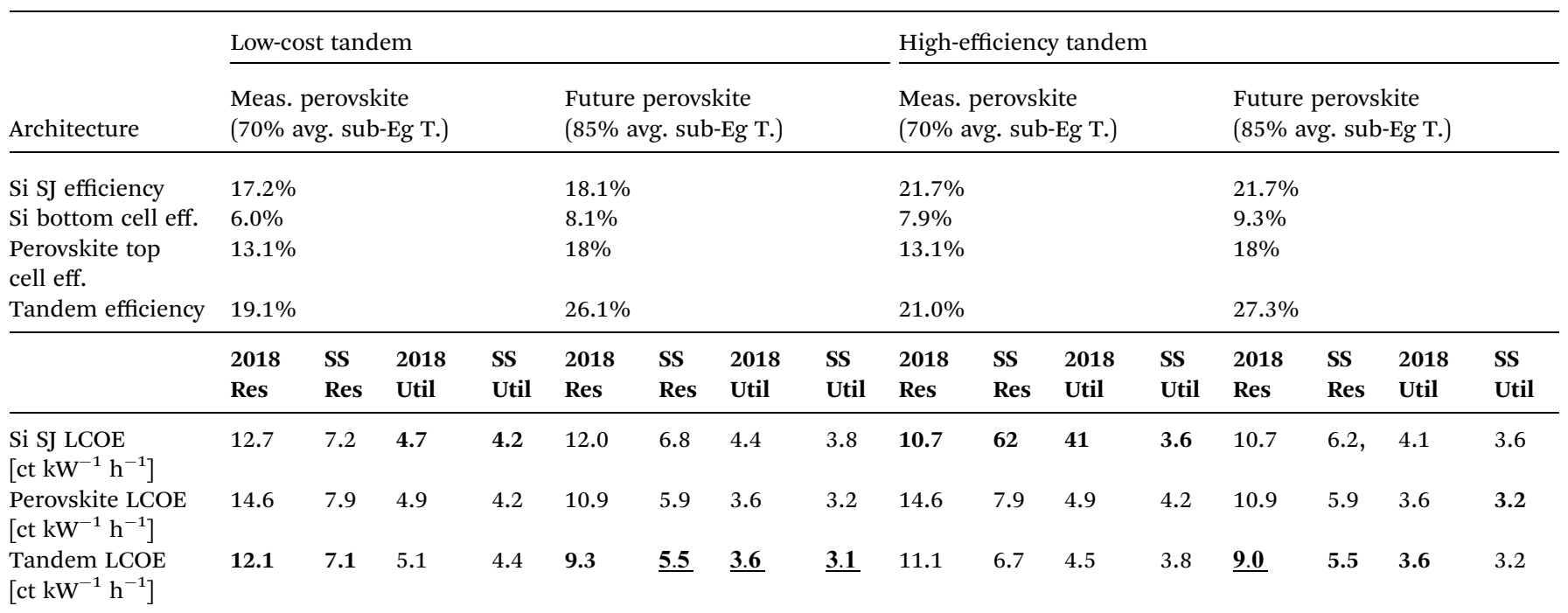


Table 4 Summary of the IV parameters and efficiencies for the measured mc-Si cell, the modeled mc-Si module efficiency based on removal of loss due to small area and glass reflection loss. The IV parameters for the record-level, large-area HIT cell from Panasonic ${ }^{27,28}$ as well as the expected module efficiency given added module loss and glass reflection. For each relevant case, the measured or modeled bottom cell and tandem efficiencies under the measured perovskite cell (13.1\% efficiency with $70 \%$ average sub-bandgap transmission), and/or the modeled perovskite top cell ( $18 \%$ efficient with $80 \%$ average sub-bandgap transmission). Note that the average sub-bandgap transmission of $70 \%$ an $8 \%$ include any glass reflection loss

\begin{tabular}{|c|c|c|c|c|c|c|c|c|}
\hline & \multirow[b]{2}{*}{$V_{\mathrm{OC}}(\mathrm{V})$} & \multirow[b]{2}{*}{$\begin{array}{l}J_{\mathrm{sc}, 1 \text { sun }} \\
\left(\mathrm{mA} \mathrm{cm}{ }^{-2}\right)\end{array}$} & \multirow[b]{2}{*}{$\mathrm{FF}(\%)$} & \multirow{2}{*}{$\begin{array}{l}\eta_{\mathrm{Si}} \\
\text { SJ } \\
\text { (\%) }\end{array}$} & \multicolumn{2}{|c|}{$\begin{array}{l}\text { Measured perov. top cell } \\
\left(\eta_{\text {perov. }}=13.1 \%, 70 \% \mathrm{~T}\right)\end{array}$} & \multicolumn{2}{|c|}{$\begin{array}{l}\text { Modeled perov. } \\
\text { top cell } \\
\left(\eta_{\text {perov. }}=18 \%,\right. \\
80 \% \mathrm{~T})\end{array}$} \\
\hline & & & & & $\eta_{\text {bot.Si }}(\%)$ & $\eta_{4 \mathrm{~T}}(\%)$ & $\begin{array}{l}\eta_{\text {bot.Si }} \\
(\%)\end{array}$ & $\begin{array}{l}\eta_{4 \mathrm{~T}} \\
(\%)\end{array}$ \\
\hline Measured mc-Si cell & 0.61 & 37.3 & 76 & 17.2 & 6.0 & 19.1 & - & - \\
\hline Modeled mc-Si mod & 0.65 & 35.0 & 79 & 18.1 & 7.2 & 20.3 & 8.1 & 26.1 \\
\hline Published c-Si cell & 0.75 & 39.5 & 83 & 24.7 & - & - & - & - \\
\hline Modeled c-Si cell & 0.75 & 36.6 & 80 & 21.9 & 9.3 & 13.1 & 10.4 & 28.4 \\
\hline
\end{tabular}

than their Si SJ but higher than the perovskite SJ. Lastly, looking at the lowest LCOE for each system scenario, the lowest LCOE device for current residential systems, among all considered, is the high-efficiency tandem using an $18 \%$ efficient top cell, however the low-cost tandem with the $18 \%$ top cell is favored in both current and future residential systems, as well as current utility systems. These results show that perovskite-silicon tandems can be viable and a tandem using a low-cost bottomcell has potential to succeed, especially as system costs continue to fall. Even in utility systems where tandems tend to be less competitive, ${ }^{13}$ the tandems with an $18 \%$ top cell reduce the LCOE compared to the silicon SJ.

\section{B. Parametric cost relations}

We then extended this analysis to explore the effect of improving top-cell efficiency by comparing the two tandems and their comprising sub-cell single-junctions' LCOEs over varying perovskite efficiency, again for utility and residential systems for 2018 and the future, reduced system cost scenario (Fig. 3). The four devices explored are shown in Fig. 1. The tandem efficiencies are modeled using the two $J V$ curve parameters for each silicon cell from the previous section and using a range of $70.5-90 \%$ average sub-bandgap transmission, the $70.5 \%$ transmission corresponding to the original measured perovskite transmission and the $90 \%$ transmission calculated by scaling the measured transmission curve (see $\mathrm{ESI}^{\dagger}$ for transmission curves). This corresponds to $7.2-9.0 \%$ efficiency from the PERC mc-Si bottom cell and 9.2-10.3\% efficiency from the HIT c-Si bottom cell. This range of sub-bandgap transmission can also represent a range in relative low-light performance or other factors causing variations in bottom cell performance.

From these results, we see that as perovskite efficiency increases, the low-cost tandem LCOE decreases more quickly than the high-efficiency tandem, causing the high-efficiency tandem to have a smaller advantage compared to the low-cost tandem. As a result, the low-cost tandem LCOE is expected to become increasingly competitive, relative to the high-efficiency tandem, as perovskite cells are developed and reach higher efficiencies. This is because an increase in efficiency becomes less valuable at higher baseline efficiencies, ${ }^{22}$ making a higher efficiency top cell reduce the benefit of an improvement in the bottom cell.

In the 2018 residential case, the high efficiency tandem is the lowest LCOE option provided the perovskite top cell efficiency is above $\sim 16 \%$ (comparing equivalent sub-bandgap transmission fractions), however the two tandem's LCOE remain quite close. For perovskite efficiencies above 16\%, the tandem LCOEs are within $1.3 \%$ and $2.4 \%$ of each other for the $70.5 \%$ and $90 \%$ transmission cases, respectively, and get closer at higher perovskite efficiencies. For the reduced system cost scenario, however, the low-cost tandem has a lower LCOE than the highefficiency tandem when the perovskite top cell efficiency is above $\sim 16 \%$, though the two tandems' LCOEs again remain close. These results suggest that general trends of improved perovskite efficiencies and continued reduction in system costs will increasingly favor the low-cost tandem over the highefficiency tandem, however their relative LCOE values do not clearly favor either tandem since they are so close. For utility scale systems, the low-cost tandem always has a lower LCOE than the high-efficiency tandem, since the lower system costs of utility scale installations value low module costs more than efficiency, compared to residential. However, as the efficiencies of perovskite SJ solar cells improve, their LCOE becomes the lowest of all architectures. Additionally, we are using a fairly high bandgap perovskite top cell in this analysis compared to many experimentally demonstrated perovskite cells. This high bandgap favors the higher efficiency tandem since the bottom cell produces a larger fraction of the tandem power with a higher bandgap top cell since more light reaches the bottom cell, thus making a high efficiency bottom cell more valuable. A lower bandgap top cell, therefore, would cause the low cost tandem to be more beneficial relative to the high-efficiency tandem.

In addition to comparing the two tandems' LCOEs directly, a key factor to consider when assessing tandems is the relative 

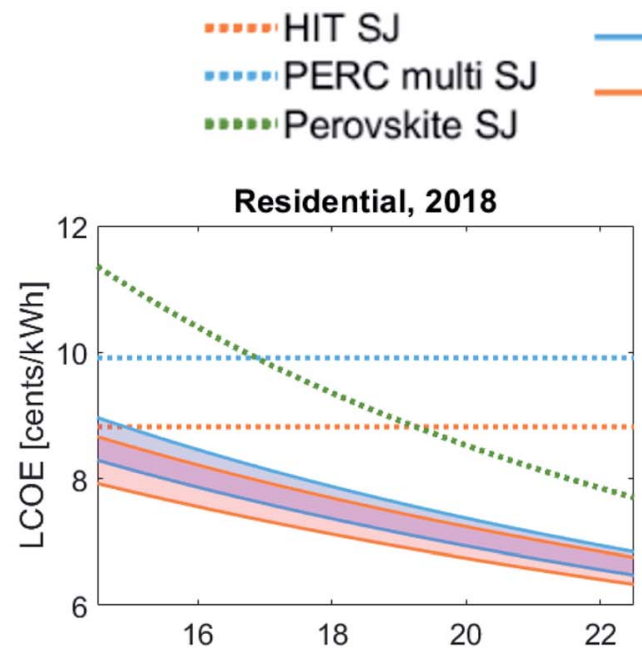

a)

Perovskite Efficiency [\%]

Utility, 2018

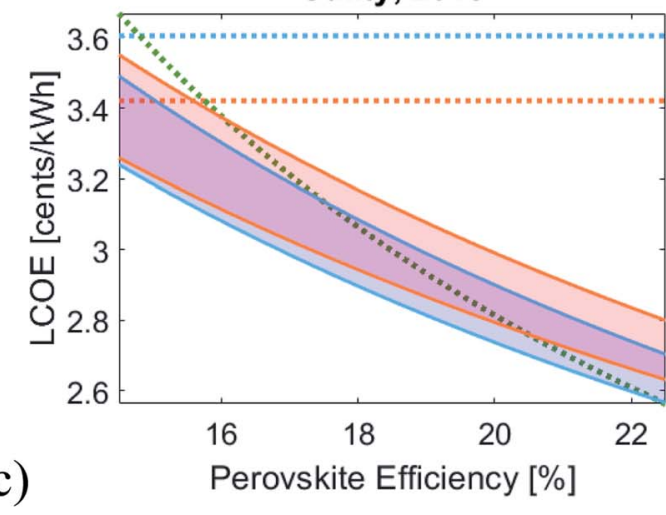

PERC multi Si-Perovskite 4T HIT Si-Perovskite 4T
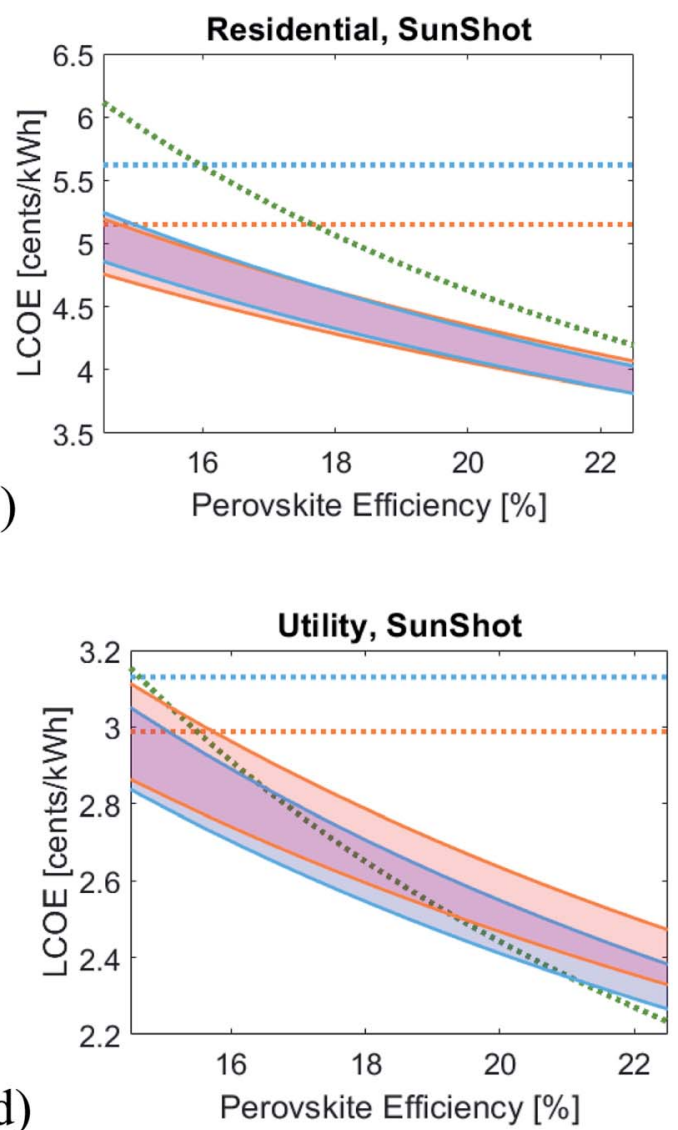

Fig. 3 LCOE of each single-junction and 4T tandem architecture versus perovskite assuming (a) 2018 residential cost, (b) reduced residential BOS cost scenario, (c) 2018 utility costs, (d) reduced utility BOS cost scenario. The two tandem LCOE values are calculated using a range of subbandgap transmission, $70.5-90 \%$.

LCOE of each tandem to its comprising sub-cells. In particular, perovskite-silicon tandems must pose a significant LCOE advantage over their comprising silicon SJ solar cell, as silicon is the established, existing technology. We see that the low-cost tandem LCOE is always lower than the low-cost Si SJ LCOE in both system cost scenarios and both system scales, while the high-efficiency tandem requires a perovskite top cell efficiency above $\sim 16 \%$ or $\sim 18 \%$ for the 2018 and reduced system cost scenarios, respectively. Furthermore, the relative LCOE benefit compared to the Si bottom cell alone is much less for the highefficiency tandem. For example, for a top cell efficiency of $18 \%$, the low-cost tandem LCOE advantage compared to the Si subcell operated independently is $15 \%$ to $20 \%$ (for the $70.5 \%$ and $90 \%$ sub-bandgap transmission cases, respectively), whereas the high-efficiency tandem LCOE advantage is only $5 \%$ to $10 \%$. This means that, though the high-efficiency tandem is costeffective compared to its sub-cells, the relative benefit is small. Thus, the investment required to develop and commercialize these tandems using high-efficiency silicon may not be worthwhile. Investment in tandem technology that utilizes low- cost, lower quality silicon bottom cells, on the other hand, provides a much greater opportunity.

We primarily use the perovskite costs based on the somewhat optimistic, forward-looking cost model from ref. 3, however, since perovskites have not yet been commercialized, there is uncertainty in their manufacturing cost. Another detailed cost model presented in ref. 29 uses a perovskite stack and fabrication process that is more conservative and predicts higher costs for the perovskite cell. To understand the impact of perovskite cell cost on our results, we find the maximum added cost of adding a perovskite top cell such that the perovskitesilicon tandem sustains a lower LCOE than the silicon bottom cell SJ alone (Fig. 4). We do this for both the high-efficiency and low-cost silicon bottom cells, across all system cost scenarios. This shows that the results for the low-cost perovskite cell are not very sensitive to perovskite cost - there is room for the perovskite to be more expensive while the tandem maintains the lowest LCOE, especially in the residential case. The highefficiency bottom cell, on the other hand, is much closer to the threshold at which an increase in the perovskite cost makes 

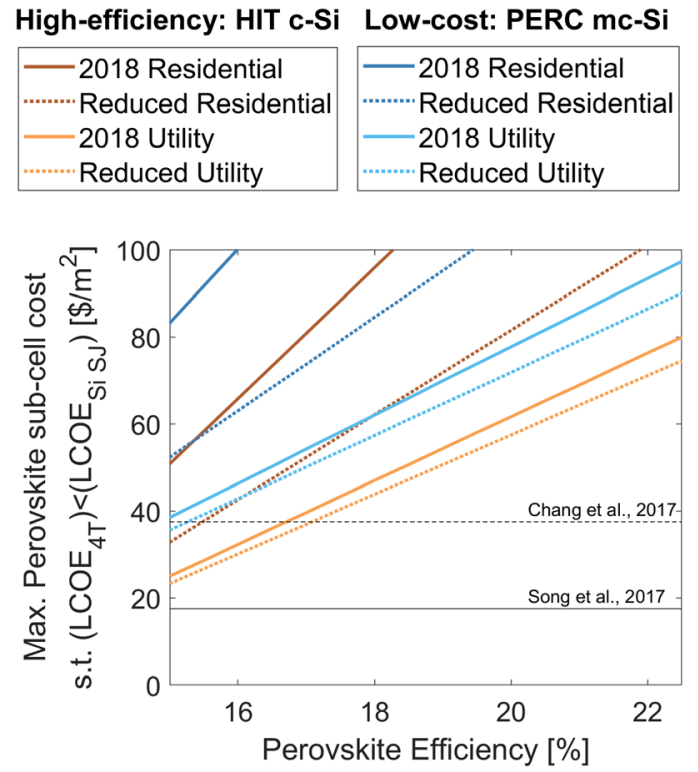

Fig. 4 Maximum added areal cost of adding the perovskite top cell to the bottom Si SJi bottom cell and the high-efficiency HIT c-Si cell in the cases of current and reduced system costs for residential and utility scale.

the tandem no-longer cost-effective, thus pushing the perovskite efficiency requirement up to about $17 \%$ for utility systems, if the costs are what is projected in ref. 4 . In both cases, the LCOE advantage relative to the silicon SJ is reduced. Conversely, as the perovskite top cell becomes more expensive, the perovskite SJ LCOE increases more quickly than the tandem, thus making the tandem more beneficial relative to the top cell perovskite.

In order to more generally understand the trade-off between the bottom cell cost and efficiency, we model the LCOE of the perovskite-silicon tandem over a varying silicon cell areal cost and bottom cell efficiency (Fig. 5). This allows us to see both the trends of LCOE with bottom cell cost and efficiency, and how changing costs and performance over time will affect the tandem favorability. We vary the energy yield linearly with efficiency, using the high-efficiency silicon bottom cell energy yield as a baseline. The perovskite top cell is assumed to have a constant efficiency of $18 \%$ and an average sub-bandgap transmission of $85 \%$. We additionally plot contour lines of the relative tandem LCOE advantage, as defined in eqn (1), colored corresponding to the magnitude of this advantage. Lastly, the HIT c-Si cell and PERC mc-Si cell are marked, along with the two corresponding 'advanced' Si cells' with reduced silicon and silver usage. ${ }^{20}$

This figure illustrates the inherent challenge that tandems face: what leads to the largest relative benefit for tandems is not necessarily aligned with what leads to the lowest LCOE. However, since an almost free silicon cell that is over $20 \%$ efficient seem unlikely, we want to instead look at what bottom cell can best be optimized for both a low LCOE and a significant relative LCOE advantage to justify the investment in development and potential associated risk.
We see two distinct trends in the relationship between LCOE reduction and relative tandem advantage for different bottom cell efficiencies regimes. For lower efficiency silicon bottom cells that fall to the left of the central diagonal of the relative LCOE advantage contour plot in Fig. 5, efficiency improvements or cost reductions of the silicon cell will both reduce LCOE and improve the relative tandem advantage. This allows tandem and bottom cell development to work synergistically. The low-cost PERC mc-Si cell falls into this regime for both current and future residential scenarios. Starting from the low-cost silicon cell parameters, both improvements in efficiency and cost reductions help reduce LCOE and improve the tandem advantage, however further reductions in cost are more advantageous than efficiency increases as they improve the tandem advantage more quickly while also reducing the LCOE directly. We see that the 'advanced Si' cost savings greatly reduces the tandem LCOE and improves the relative tandem advantage. Additionally, there is room for the efficiency to fall with cost while still reducing the tandem LCOE and increasing the tandem advantage. For all scenarios except current residential, for a constant $\$$ per $\mathrm{W}$ equal to the low-cost silicon cell, reducing the cost and efficiency of the bottom cell improves the tandem LCOE and the tandem advantage. Efficiency gains for the low-cost cell will also reduce LCOE, but will only marginally increase (or potentially decrease, depending on the magnitude of the efficiency increase) the tandem advantage.

For higher efficiency bottom cells that fall to the right of the central diagonal of the relative LCOE advantage contour plot, increasing efficiency or reducing the cost of the silicon cell always reduces the tandem LCOE but also reduces the tandem LCOE advantage, making the tandem less desirable compared to its subcells. This is because in this regime, the silicon cell has a lower LCOE than the perovskite SJ, so the tandem must compete with the silicon SJ LCOE. Improvements in the silicon bottom cell, however, benefit the SJ more than the tandem. The high efficiency HIT c-Si cell falls into this regime for the 2018 residential case and on the edge of this regime in the future residential scenario. This means that as the high-efficiency silicon cell's cost decreases or efficiency improves, the high-efficiency tandem becomes less attractive because the Si SJ LCOE will fall more quickly than the tandem LCOE. For this to not be the case, a much better perovskite top cell would be required (but this may again cause the perovskite cell to then be more economically viable than the high-efficiency silicon single junction). Furthermore, the we see that the 'advanced' multi- or monocrystalline silicon cells improves the LCOE advantage of the low-cost tandem over the high-efficiency tandem. This enforces that expected market directions further advantage the low-cost tandem.

For utility scale, both silicon cells are too expensive for the tandem to have the lowest LCOE. If the cost of the low-cost, mc-Si PERC cell is reduced by even a small margin, which can be achieved through kerfless wafers, however, the tandem will have a lower LCOE than either SJ solar cell. This is also true for the high-efficiency c-Si HIT cell, however the LCOE of the tandem and the silicon SJ would be very close, giving tandems little advantage. 


\begin{tabular}{llllll}
\hline high-efficiency HIT c-Si $\quad \bigcirc \quad$ low-cost PERC mc-Si $\quad \times \quad$ kerfless low-cost $\quad \times \quad$ kerfless high-eff. \\
\hline
\end{tabular}

Residential, 2018

Relative Tandem LCOE advantage

$\begin{array}{lllll}4.74 & 9.92 & 15.09 & 20.27 & 25.45\end{array}$

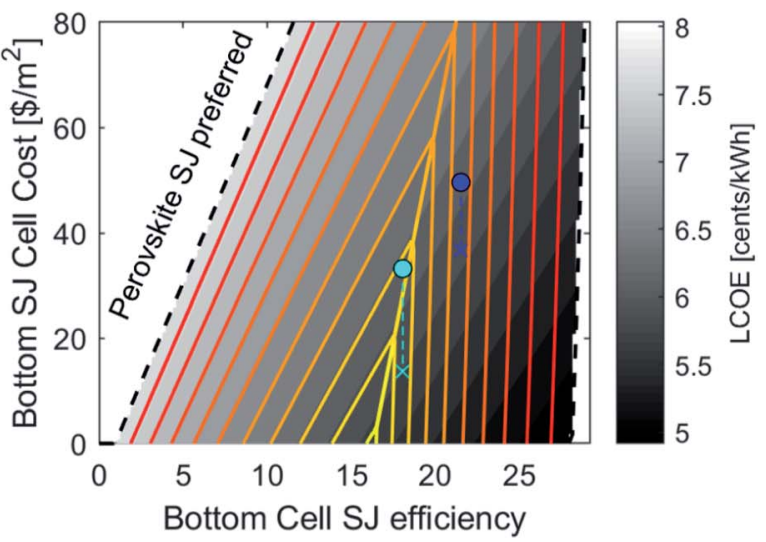

Utility, 2018

Relative Tandem LCOE advantage $\begin{array}{lllll}1.53 & 6.25 & 10.96 & 15.68 & 20.4\end{array}$

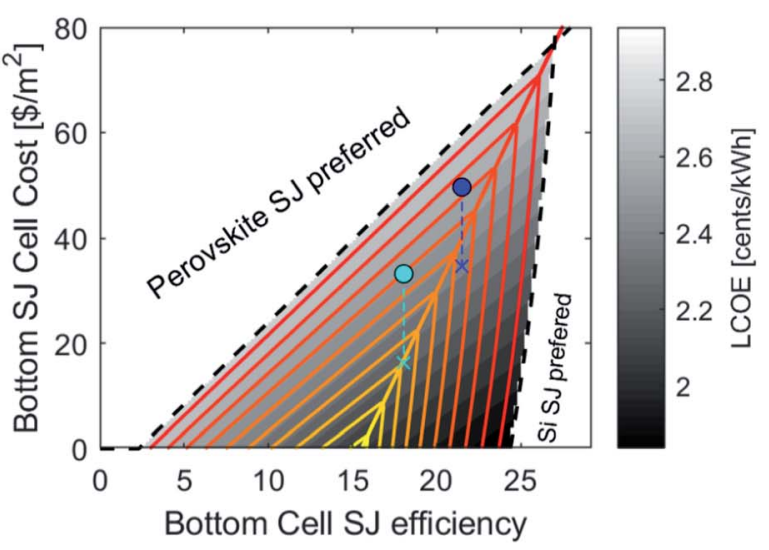

Residential, RSCS

Relative Tandem LCOE advantage

$\begin{array}{lllll}2.16 & 6.86 & 11.57 & 16.27 & 20.98\end{array}$

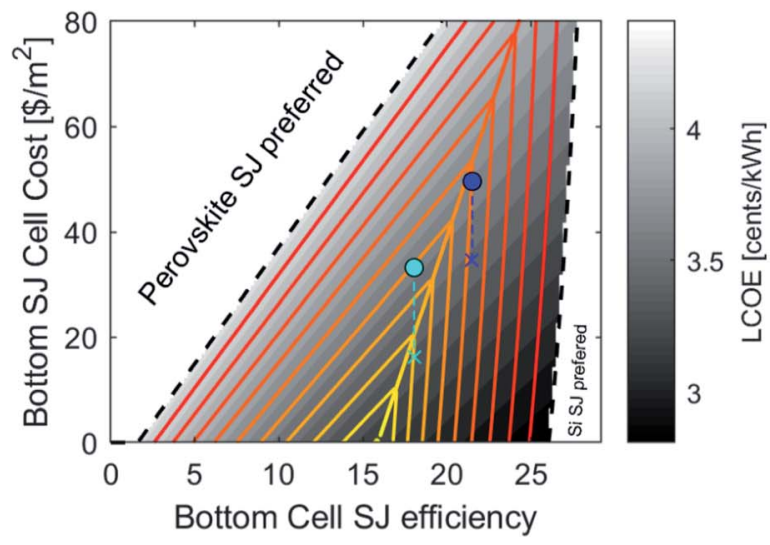

Utility, RSCS

Relative Tandem LCOE advantage $\begin{array}{lllll}2.15 & 6.78 & 11.41 & 16.04 & 20.67\end{array}$

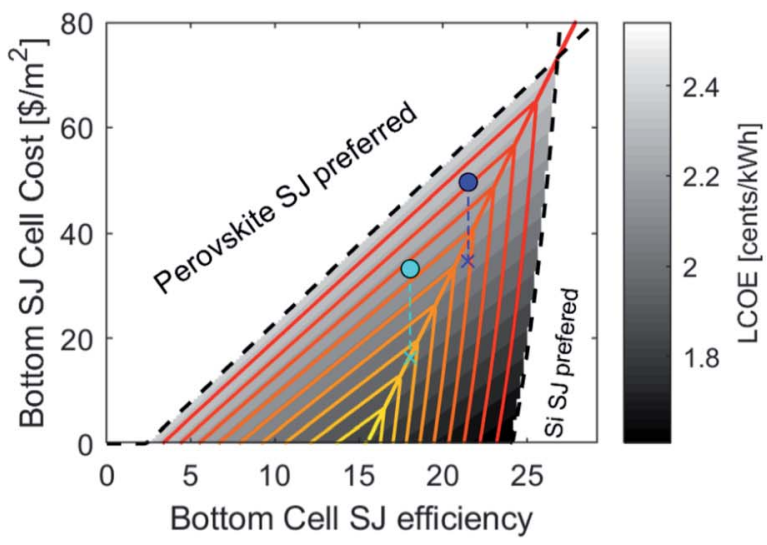

Fig. 5 The grey contour plot shows the LCOE of a perovskite-silicon tandem over a range of bottom cell single-junction efficiencies and areal costs, assuming an $18 \%$ perovskite top cell with average sub-bandgap transmission of $85 \%$. The red-to-yellow lines trace contour lines of the relative $L C O E$ advantage, i.e. $\frac{\min \left(\mathrm{LCOE}_{\mathrm{Si}}, \mathrm{LCOE}_{\text {perov. }}\right)-\mathrm{LCOE}_{4 \mathrm{~T}}}{\min \left(\mathrm{LCOE}_{\mathrm{Si}}, \mathrm{LCOE} E_{\text {perov. }}\right)}$. The regions left white indicate where one of the single-junction sub-cells (noted by the overlaid text) has a lower LCOE than the tandem. The low-cost and high-efficiency cells are marked, along with an ' $x$ ' indicating the cost 'advanced' multi- or monocrystalline silicon cells are used, respectively, with reduced silicon and silver usage.

\section{Considerations for investing in tandems}

From the perspective of a silicon manufacturer that produces high-efficiency silicon modules, investing in perovskite tandems is likely not worthwhile as the benefit will be small and requires perovskite cell efficiency above $16 \%$ to maintain any benefit as system costs fall. If the efficiency of the perovskite top-cell on a module level reaches $20 \%$, tandems look more appealing since the relative tandem LCOE advantage would exceed $15 \%$, however, at this point, perovskite single-junctions will likely be more viable then either the tandem or the silicon SJ. Furthermore, any improvement of the silicon cell's efficiency will make the relative tandem LCOE even less beneficial. If the Si-SJ module efficiency reaches $\sim 25 \%$, then the tandem and SiSJ LCOE will be equivalent, assuming an $18 \%$ perovskite top cell efficiency and unchanged Si manufacturing cost. This parityachieving efficiency decreases as the silicon manufacturing cost and system costs decrease.

From the perspective of a manufacturer of lower cost multicrystalline silicon solar cells, investing in perovskites has the 
potential to be greatly beneficial by reducing the LCOE of their product and opening up opportunities to compete in the residential market - an area of the solar market that multicrystalline silicon solar cells currently cannot compete in because of their low efficiency. The relative benefit of the tandem is fairly robust to potential uncertainty in perovskite cell manufacturing cost and efficiency, as well as to expected trends in system costs. Furthermore, adoption of technological advancements to lower costs such as reducing silicon and silver usage will increase the benefit of the tandem and the relative advantage over the high-efficiency tandem. The design of the bottom cell can also be optimized based on lower expected current-carrying requirements, making series resistance less important. Such reductions in multicrystalline silicon cells manufacture could enable these tandems to compete with the perovskite SJ in utility scale applications.

Lastly, from the perspective of the broader solar community and in the interest of reducing the LCOE of solar electricity generally, we see that both tandems provide a path to greatly reduced LCOE compared to the silicon single-junction technology, decreasing LCOE by $9-13 \%$ (depending on the scenario), compared to the lowest LCOE Si SJ and assuming an $18 \%$ efficient perovskite top-cell. The two tandems suggest similar resulting LCOEs, thus neither seems to obviously be more viable, however expect directions in the solar market, changes in manufacturing, and efficiency improvement of both sub-cells indicate the lowcost tandem will more easily achieve a lower LCOE.

Additionally, the multicrystalline silicon bottom cells have a large CapEx advantage which allows for much faster growth in manufacturing capacity. ${ }^{30}$ Thus, low-cost silicon-perovskite tandems could enable a faster growing solar market - a necessity for near term terawatt scale installation of solar panels. ${ }^{31}$ Furthermore, the low-cost silicon CapEx and growth rate will more closely match the CapEx and growth rate of perovskite solar cells than the high-efficiency silicon, making multicrystalline silicon and perovskite a better pairing.

\section{Conclusion}

In this work, we perform techno-economic analysis to explore the trade-off of cost-versus-efficiency for silicon bottom cells in a perovskite-silicon tandem in order to define the optimal approach to make cost-effective perovskite-silicon tandems. We show that perovskite-silicon tandems can be made costeffective, competitive, and provide sufficient benefits for investment by using current, available low-cost multicrystalline silicon technology, with further advantages from even lower cost kerfless wafer production. Furthermore, these tandems are robust to and benefit from expected technological advances and market trends. Transitioning silicon manufacturing to highefficiency, high-cost architectures for use in tandems is shown to be in unnecessary and potentially disadvantageous for achieving competitive tandems that reduce LCOE.

We fabricate a perovskite-silicon tandem with a multicrystalline bottom cell to demonstrate the viability of using a low-cost silicon bottom cell. Our tandem reaches an efficiency of $19.1 \%$, surpassing the values for either comprising sub-cell operated independently. We then compare, more generally through modeling, perovskite-silicon four-terminal tandems that employ a high-efficiency, high-cost silicon bottom cell versus tandems with low-cost and lower-efficiency bottom cell. The figure of merit for this comparison is the LCOE for utility and residential scale installations for both current and future system cost scenarios. Through broader parametric cost analysis, we see that a low-cost mc-Si based tandem has great potential to reduce LCOE relative to the mc-Si single-junction, and possibly open mc-Si technology to new, more lucrative markets like high-efficiency rooftop markets.

In current residential systems, the high-efficiency tandem has a lower LCOE than the low-cost tandem, yet that advantage is small and, as system costs continue to fall, is lost entirely. The low-cost tandem has a lower LCOE than the high-efficiency tandem for utility systems. In all cases, as perovskite top-cell efficiency improves, the low-cost tandem has an increasingly competitive LCOE compared to the high-efficiency tandem. Furthermore, the low-cost tandem has a significantly lower LCOE than the low-cost silicon bottom cell, demonstrating a clear benefit to invest in transitioning to tandem technology, while the tandem using the high-efficiency silicon cell has an LCOE that is close to or potentially higher than the LCOE of the high-efficiency silicon single-junction cell. This demonstrates that there is a compelling argument to pursue perovskite-silicon tandems that employ low-cost, lower-efficiency bottom cells as the benefit of the tandem is more significant and more certain. Lastly, we map out a path towards optimizing for both device LCOE reduction and the relative benefit of a tandem compared to its sub-cells. A tandem using a high-cost, high-efficiency bottom cell, despite achieving a higher efficiency, is likely not economically favorable compared to its sub-cells or compared to the low-cost tandem. This finding stands in contrast with much of the perovskite-silicon tandem device development and motivates research to explore lower-cost, lower-efficiency silicon bottom cells for tandems that may not achieve record efficiencies but are likely to be more commercially viable.

\section{Conflicts of interest}

There are no conflicts to declare.

\section{Acknowledgements}

The authors thank Dr Kevin Bush for contributing cells early on for this work and Dr Adam Lorentz for his support and feedback. This work was financially supported by the DOE-NSF ERF for Quantum Energy and Sustainable Solar Technologies (QESST), by funding from Singapore's National Research Foundation through the Singapore MIT Alliance for Research and Technology's "Low energy electronic systems (LEES) IRG", and the funding from the Martin Family Society of Fellows for Sustainability.

\section{References}

1 D. M. Powell, M. T. Winkler, A. Goodrich and T. Buonassisi, Modeling the cost and minimum sustainable price of 
crystalline silicon photovoltaic manufacturing in the United States, IEEE J. Photovolt., 2013, 3(2), 662-668.

2 R. Jones-Albertus, D. Feldman, R. Fu, K. Horowitz and M. Woodhouse, Technology advances needed for photovoltaics to achieve widespread grid price parity, Prog. Photovoltaics, 2016, 24(9), 1272-1283.

3 Z. Song, et al., A technoeconomic analysis of perovskite solar module manufacturing with low-cost materials and techniques, Energy Environ. Sci., 2017, 10(6), 1297-1305.

4 N. L. Chang, A. W. Y. Ho-Baillie, P. A. Basore, T. L. Young, R. Evans and R. J. Egan, A manufacturing cost estimation method with uncertainty analysis and its application to perovskite on glass photovoltaic modules, Prog. Photovoltaics, 2017, 25(5), 390-405.

5 I. M. Peters, S. Sofia, J. Mailoa and T. Buonassisi, Technoeconomic analysis of tandem photovoltaic systems, RSC Adv., 2016, 6(71), 66911-66923.

6 Z. J. Yu, J. V. Carpenter and Z. C. Holman, Techno-economic viability of silicon-based tandem photovoltaic modules in the United States, Nat. Energy, 2018, 3, 747-753.

7 T. Duong, et al., Rubidium Multication Perovskite with Optimized Bandgap for Perovskite-Silicon Tandem with over 26\% Efficiency, Adv. Energy Mater., 2017, 1700228, 1-11.

8 F. Sahli, et al., Fully textured monolithic perovskite/silicon tandem solar cells with $25.2 \%$ power conversion efficiency, Nat. Mater., 2018, 17(9), 820-826.

9 Oxford PV perovskite solar cell achieves 28\% efficiency, Oxford PV, https:/www.oxfordpv.com/news/oxford-pv-perovskitesolar-cell-achieves-28-efficiency.

10 Photovoltaics report, Prepared by Fraunhofer Institute for Solar Energy Systems, ISE with support of PSE Conferences \& Consulting $\mathrm{GmbH}, 2018$.

11 E. Bellini, 1366 Technologies and Hanwha Q Cells to open wafer factory in Malaysia, PV Magazine, Feb-2019.

12 J. P. Mailoa, M. Lee, I. M. Peters, T. Buonassisi, A. Panchula and D. Weiss, Energy-Yield Prediction for II-VI-Based ThinFilm Tandem Solar Cells, Energy Environ. Sci., 2016, 9, 2644-2653.

13 S. E. Sofia, J. P. Mailoa, D. N. Weiss, B. J. Stanbery, T. Buonassisi and I. M. Peters, Economic viability of thinfilm tandem solar modules in the United States, Nat. Energy, 2018, 3, 387-394.

14 C. A. Gueymard, SMARTS, A Simple Model of the Atmospheric Radiative Transfer of Sunshine: Algorithms and Performance Assessment, Florida Solar Energy Center, Clearlake Rd., Cocoa, FL, 1995, p. 32922, Professional Paper FSEC-PF-27095.

15 C. Gueymard, Parameterized Transmittance Model for Direct Beam and Circumsolar Spectral Irradiance, Sol. Energy, 2001, 71(5), 325-346.
16 System Advisory Model (SAM): Financial Model Documentation, 2010, https:/sam.nrel.gov/financial, accessed: 09-Jan-2017.

17 D. C. Jordan and S. R. Kurtz, Photovoltaic Degradation Rates - An Analytical Review, Prog. Photovoltaics, 2013, 21, 12-29.

$18 \mathrm{~L}$. Meng and J. You, Addressing the stability issue of perovskite solar cells for commercial applications, Nat. Commun., 2018, 1-4.

19 G. Grancini, et al., One-Year stable perovskite solar cells by 2D/3D interface engineering, Nat. Commun., 2017, 8, 15684.

20 M. Woodhouse, B. Smith, A. Ramdas, and R. Margolis, Crystalline Silicon Photovoltaic Module Manufacturing Costs and Sustainable Pricing: $1 H 2018$ Benchmark and Cost Reduction Roadmap, 2019.

21 R. Fu, D. Feldman, and R. Margolis, U.S. Solar Photovoltaic System Cost Benchmark: Q1 2018, 2018.

22 A. Goodrich, T. James, and M. Woodhouse, Residential, Commercial, and Utility-Scale Photovoltaic (PV) System Prices in the United States: Current Drivers and Cost-Reduction Opportunities, NREL/TP-6A20-53347, 2012.

23 R. Jones-albertus, D. Feldman, R. Fu, K. Horowitz and M. Woodhouse, Technology Advances Needed for Photovoltaics to Achieve Widespread Grid Price Parity, Prog. Photovolt: Res. Appl., 2016, 24, 1272-1283.

24 T. Duong, et al., Rubidium Multication Perovskite with Optimized Bandgap for Perovskite-Silicon Tandem with over 26\% Efficiency, Adv. Energy Mater., 2017, 7, 1700228.

25 W. S. Yang, et al., High-performance photovoltaic perovskite layers fabricated through intramolecular exchange, Science, 2015, 348(6240), 2013-2017.

$26 \mathrm{~J}$. P. Correa-Baena, et al., The rapid evolution of highly efficient perovskite solar cells, Energy Environ. Sci., 2017, 10(3), 710-727.

27 M. Taguchi, et al., 24.7\% Record efficiency HIT solar cell on thin silicon wafer, IEEE J. Photovolt., 2014, 4(1), 96-99.

28 M. A. Green, K. Emery, Y. Hishikawa, W. Warta and E. D. Dunlop, Solar cell efficiency tables (version 42), Prog. Photovoltaics, 2013, 21, 827-837.

29 A. D. Mills and R. H. Wiser, Strategies to mitigate declines in the economic value of wind and solar at high penetration in California, Appl. Energy, 2015, 147, 269-278.

30 D. M. Powell, R. Fu, K. Horowitz, P. A. Basore, M. Woodhouse and T. Buonassisi, The capital intensity of photovoltaics manufacturing: barrier to scale and opportunity for innovation, Energy Environ. Sci., 2015, 8(12), 3395-3408.

31 D. Berney Needleman, J. R. Poindexter, R. C. Kurchin, I. M. Peters, G. Wilson and T. Buonassisi, Economically Sustainable Scaling of Photovoltaics to Meet Climate Targets, Energy Environ. Sci., 2016, 9, 2122-2129. 